\title{
Letter identification declines with increasing retinal eccentricity at the same rate for normal and dyslexic readers
}

\author{
RAYMOND KLEIN, GLEN BERRY, KEVIN BRIAND, BARBARA D'ENTREMONT, \\ and MARY FARMER \\ Dalhousie University, Halifax, Nova Scotia, Canada
}

\begin{abstract}
It has recently been claimed (Geiger \& Lettvin, 1987; Perry, Dember, Warm, \& Sacks, 1989) that the acuity/eccentricity function is flatter in dyslexics than in normal subjects, with dyslexics showing better performance in the periphery and worse performance at fixation. In these studies, all target letters were presented to the right of fixation, a procedural flaw inviting subjects to optimize performance by directing attention and/or gaze to the right of the designated fixation point. It is suggested that dyslexic and normal readers may differ in the degree to which they might adopt the optimal strategy in this situation. To overcome this problem, target letters were briefly presented at 16 randomly intermixed locations derived from the orthogonal combination of four eccentricities and four directions from fixation (above, below, right, left). The accuracy of letter identification declined with increasing eccentricity at the same rate for good and poor adult readers and dyslexic teenagers. This finding provides no support for the view that the acuity/eccentricity function might vary with and possibly cause differences in reading level.
\end{abstract}

Support for the proposition that the quality of visual information for form recognition declines with increasing retinal eccentricity goes back at least to 1857 , when Aubert and Foerster (cited by Geiger \& Lettvin, 1987) explored the identification of letters of various sizes at various retinal eccentricities. They found that as the retinal eccentricity of a letter was increased, it had to be made larger to be just identifiable. Accordingly, they also demonstrated that for letters of a given size, identification accuracy declined with increasing retinal eccentricity, a result that has since been replicated many times (see, e.g., Woodworth \& Schlosberg, 1954, pp. 103-104). Today it is taken for granted that the shape of the Aubert-Foerster function is mediated by the density of neural representation which, at both retinal and cortical levels, favors central over peripheral vision (Hughes, 1977).

In a recent paper, Geiger and Lettvin (1987) reported that they had measured the function relating acuity to eccentricity in normal and dyslexic readers, and had found striking differences. In their paradigm, two letters were presented simultaneously, one at fixation and one at various eccentricities. The subject's task was to report both letters, and the dependent variable was percent correct letter identification as a function of eccentricity. The dyslexics performed better than normals at the more peripheral locations and worse more centrally (see Figure 1A), which led the authors to conclude that "the dyslexic subjects had a markedly wider area in which correct iden-

The research reported here was supported by a grant from the Natural Science and Engineering Research Council of Canada to R. Klein. Requests for reprints should be sent to R. Klein, Department of Psychology, Dalhousie University, Halifax, Nova Scotia B3H 4J1, Canada. tification occurred in the peripheral field than did the normal readers." Geiger and Lettvin also made controversial claims about masking, foveal vision, and remediation strategies. In the present investigation, we focused on the shape of the acuity/eccentricity function for single letters. Geiger and Lettvin argued that the relatively improved processing of peripheral information by dyslexics might mediate their poor reading via increased masking of the fixated material.

The Geiger and Lettvin study has received considerable attention, and generated considerable commotion (see, e.g., letters to the editor published in the New England Joumal of Medicine, 1987, 317, pp. 1737-1739). At the 1988 meeting of the Psychonomic Society, Perry, Dember, Warm, and Sacks (1988) identified some of the reasons for this intense interest:

The news media and the public they serve seem to latch onto each new study purporting to have identified the way in which dyslexic individuals differ from normal readers beyond the obvious difference in reading ability itself. A recent case in point is an article published by Gad Geiger and Jerome Lettvin.... As with any clinical entity, one would expect to find some deficit underlying dyslexics' poor reading performance, perhaps a structural abnormality or some lack in ability to process perceptual or linguistic material. What may have lent special appeal to the GeigerLettvin article was their finding what appears to be a visual advantage for dyslexic readers in their ability to identify letters presented eccentrically from fixation.

Our theme is that Geiger and Lettvin's methodology is flawed, and that their finding could easily be due to differences in gaze direction or in the distribution of attention (see also Shaywitz \& Waxman, 1987). Actually, this was 
$1 \mathrm{~A}$

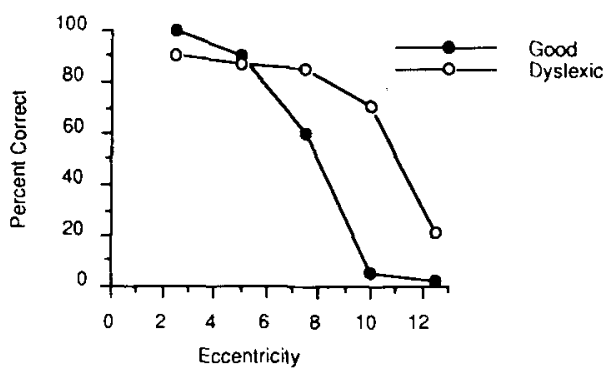

$1 \mathrm{~B}$

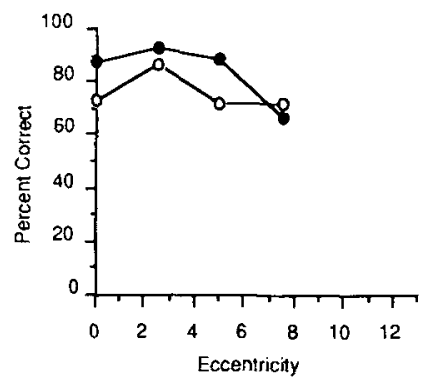

$1 \mathrm{C}$

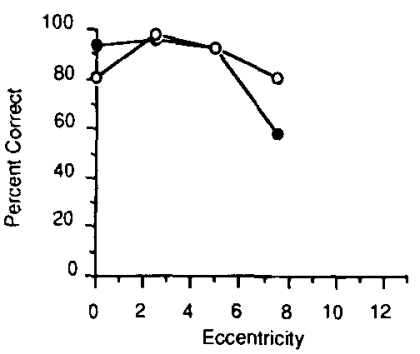

Figure 1. Identification accuracy of normal and dyslexic readers as a function of eccentricity from (A) Geiger and Lettvin (1987), dual letter task, (B) Perry et al. (1988, Experiment 1), dual letter task, and (C) Perry et al. (1988, Experiment 2), single letter task. The data are redrawn from the original sources. See text for explanation.

partly Perry et al.'s $(1988,1989)$ theme too, but many of our criticisms of Geiger and Lettvin's methodology apply equally to their replication.

In the Geiger and Lettvin (1987) study, each trial began with a fixation point that was replaced by one letter while, simultaneously, another letter appeared at a fixed eccentricity to the right of fixation. The subjects were instructed to report both letters. There are several problems with this method:

1. Subjects may differ in the amounts of attention paid to the central as opposed to the peripheral letters. Geiger and Lettvin did not provide data on the report of the foveal letters, so this type of strategy difference cannot be assessed.

2. Since all peripheral stimuli were to the right of the fixation stimulus, the subjects might have chosen to attend to the right of the fixation stimulus, thus improving overall performance; such a tendency might vary with reading level. Such an attentional shift might be accom- plished, covertly (Posner, 1980) or overtly, through an adjustment in gaze direction.

3. Moreover, since eccentricity was blocked, the subjects may have engaged in specific strategies to accommodate each eccentricity, for example, fixating or attending to the peripheral target location.

4. It is unlikely that the technique used by Geiger and Lettvin (1987) to monitor eye position (visual inspection of the subject's eyes by the experimenter) was sensitive enough to determine the degree to which the subjects were following the fixation instruction which, according to 2 and 3 above, they had reason to ignore.

In their first experiment, Perry et al. $(1988,1989)$ randomized eccentricity of the parafoveal stimulus within the right visual field and provided data on the foveal letters, thus eliminating some of these problems. Although the data from this manipulation (see Figure 1B) differ from Geiger and Lettvin's in several respects, they do reveal flatter functions for the poor readers. Perry et al. (1988) correctly noted that this pattern of results might have more to do with divided attention (between central and peripheral stimulation) than with the Aubert/Foerster function. Recall that in Geiger and Lettvin (1987) and Perry et al. (1988, 1989, Experiment 1) two letters were presented simultaneously and report of both was required. In such a situation, subjects can exert strategic control in two simple ways to effect performance tradeoffs: (1) They can vary the order of report, with performance generally being better for the first reported item; and (2) they can vary the allocation of visual attention among the possible target locations. While differences between dyslexic and normal readers in either of these two strategies would require some explanation, either would be quite different from that suggested by Geiger and Lettvin (and might require quite different remedial techniques if the strategies could be shown to be instrumentally related to the reading deficit).

In a second experiment, Perry et al. $(1988,1989)$ eliminated the central letters, thus overcoming an objection based on divided attention. Their results from this experiment (Figure 1C) closely parallel those of Geiger and Lettvin (1987) for eccentricities between $2.5^{\circ}$ and $7.5^{\circ}$. Perry et al. (1989) state that "the results of both experiments reinforce Geiger and Lettvin's (1987) conclusion that the Aubert-Foerster function is flatter for dyslexic than for normal readers. Moreover, the changes that these experiments introduced into the Geiger and Lettvin procedure add generality to that conclusion" (p. 447). We agree that the Geiger and Lettvin pattern was replicated. We strongly disagree, however, with the conclusion that dyslexics and normals have different Aubert-Foerster functions.

It seemed to us that with all the letters' occurring to the right of fixation in these studies, the optimal strategy would be to fixate in the middle of the region where letters could occur (at $3.75^{\circ}$ ) rather than at the provided fixation point. If the poor readers did that while the good readers were a little suboptimal (more obliging), then it is possible to quite closely simulate the data obtained by 
Perry et al. (1988, 1989, Experiment 2). ${ }^{1}$ Even if the subjects were following the fixation instructions, it is still possible that they were shifting attention (Posner, 1980) to the center of the region where the letters were to appear.

\section{EXPERIMENT 1}

One straightforward way to provide the subject with incentive to maintain fixation and to keep attention centered at fixation is to place the fixation point at the center of the possible target locations. ${ }^{2} \mathrm{We}$ did this by randomly selecting both the eccentricity and direction (up, down, left, right) of the target stimuli. Using this procedure in Experiment 1, we tested two groups of college students (good and poor readers).

\section{Method}

Subjects. The subjects were 14 undergraduate psychology students from Dalhousie University who received either credit points or $\$ 5$ for their participation in the study. They were selected according to their scores on a previously administered Nelson-Denny reading test (Form D, Comprehension). The scores of the good readers (4 males and 4 females) ranged from the 80 th to the 98 th percentile. The scores of the poor readers ( 2 males and 4 females) ranged from the 14 th to the 33 rd percentile. All subjects had normal or corrected-to-normal vision. Prior to the experimental session described below, the Vocabulary and Block Design subscales of the Wechsler Adult Intelligence Scale (WAIS) were administered. These subscales were chosen because they are the best predictors of verbal and performance IQ (Wechsler, 1944). Means, standard deviations, and ranges for age, IQ, and reading level are given in Table 1.

Apparatus and Stimuli. The experiment was controlled by an Apple II Plus computer equipped with a millisecond clock (and circuitry to permit timing of events to coordinate with the video synchronization pulse; Reed, 1979). The subjects were seated, with their chins on a chinrest, at a distance of about $35 \mathrm{~cm}$ from the viewing screen.

The stimuli used by Geiger and Lettvin (1987) (uppercase letters $\mathrm{C}, \mathrm{H}, \mathrm{I}, \mathrm{N}, \mathrm{O}, \mathrm{W}, \mathrm{S}, \mathrm{T}, \mathrm{E}$, and $\mathrm{Y}$ ) were presented as black letters on a white screen. The letters subtended visual angles of about $0.63^{\circ}$ horizontal and $0.85^{\circ}$ vertical. A fixation cross, which subtended a visual angle of $0.63^{\circ}$ square, was presented in the center of the viewing screen. On each trial, one letter was displayed briefly for

\section{Table 1}

Means, Standard Deviations, and Ranges for Age, Reading Level (Nelson Denny), and $1 Q$ (Block Design and

Vocabulary Subscales of the WAIS) of Good and Poor Readers Used in Experiment 1

\begin{tabular}{lccr}
\hline & $M$ & $S D$ & Range \\
\hline & Good Readers & & \\
Age & 19 & 1.30 & $18-22$ \\
Reading level & 28 & 2.27 & $25-31$ \\
IQ (WAIS): & & & \\
$\quad$ Performance* & 123 & 24.39 & $89-150$ \\
$\quad$ Verbal $\dagger$ & 122 & 10.32 & $100-129$ \\
& Poor Readers & & \\
Age & 19 & 1.37 & $18-21$ \\
Reading level & 15 & 1.33 & $14-17$ \\
IQ (WAIS): & & & \\
$\quad$ Performance* & 132 & 9.65 & $120-143$ \\
$\quad$ Verbal $\dagger$ & 99 & 9.71 & $88-114$ \\
\hline *Block Design subscale. & + Vocabulary subscale.
\end{tabular}

*Block Design subscale. †Vocabulary subscale.

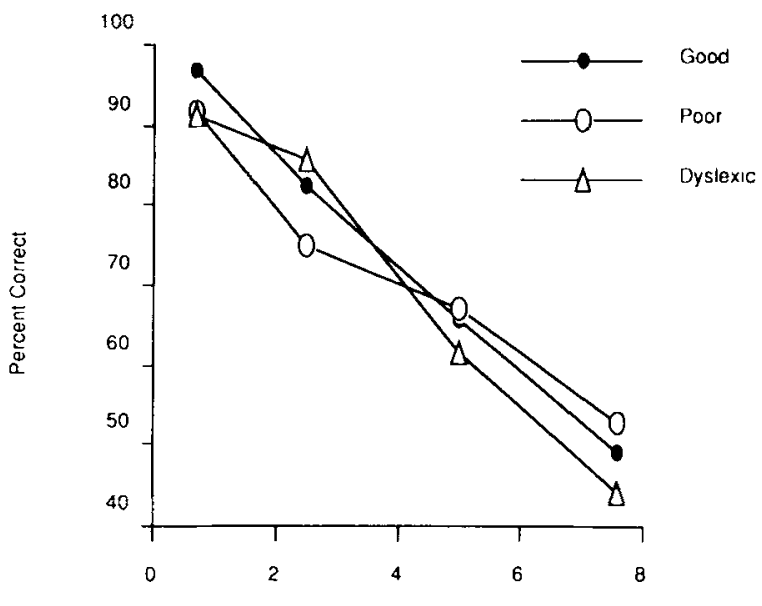

Eccentricity

Figure 2. Identification accuracy of good (closed circles) and poor (open circles) college age readers (Experiment 1) and adolescent dyslexics (open triangles; Experiment 2) as a function of eccentricity of a single letter, with direction (left, right, up, down) and eccentricity varied randomly.

one refresh cycle $(60 \mathrm{~Hz})$. The letter could appear above, below, to the left of, or to the right of the fixation cross, which remained on the screen for the duration of the experiment. In addition to the four directions, the target letter could appear at any of four possible eccentricities. The center-to-center distances from the fixation to the target at the four possible eccentricities were $0.7^{\circ}, 2.5^{\circ}, 5.0^{\circ}$ and $7.6^{\circ}$. Thus, there were 16 possible target locations. The target letter and position were selected randomly. The keyboard console was used both to initiate each trial and to collect responses.

Procedure. At the beginning of the experiment, the subjects were shown, on the screen, a display consisting of the 10 possible target letters in the 16 possible positions. The subjects were then given 20 practice trials before starting the main block of 80 trials. For each trial, the target letter, response, target position, and eccentricity were recorded. The subjects were told that they were not being timed, but that response accuracy was being measured.

Each trial began with a black fixation cross in the center of a blank white screen. The subjects pressed a spacebar to initiate each trial. After a 1-sec delay, the target stimulus appeared briefly $(17 \mathrm{msec})$ in one of the 16 possible locations. The subjects were instructed to identify the target letter by pressing the corresponding letter key on the console. A short, 200-msec tone presented immediately after the response indicated that a response had been recorded.

\section{Results and Discussion}

Identification accuracy was subjected to an analysis of variance (ANOVA), with reading level as a betweensubjects factor and eccentricity and direction as withinsubjects factors. ${ }^{3}$ The major finding (shown in Figure 2) was a significant effect of eccentricity $[F(3,36)=72.33$, $p<.001]$ that did not interact with reading level $[F(3,36)=$ 1.51]. There was a significant effect of direction $[F(3,36)=$ $9.72, p<.001$ ], with more accurate performance overall on the left and right stimuli. Direction also interacted with distance $[F(9,108)=6.3, p<.001]$ : the falloff in accuracy with increasing eccentricity was much less steep for stimuli to the left of fixation (see Table 2). Although 
the three-way interaction between reading level, direction (up, down, left, right), and eccentricity was not significant $[F(9,108)=.97]$, there appears to be a trend in Table 2 for the function to be flatter for the poor readers than for the good readers when stimuli are presented to the right of fixation (but not for the other three directions). Since this is the empirical pattern reported by Geiger and Lettvin (1987) and Perry et al. (1989), we thought it warranted closer scrutiny to determine if it was a reliable trend in our study. The interaction between reading level, direction (left/right), and eccentricity was not significant $[F(3,36)=.62]$ when the up and down directions were excluded from the analysis; the interaction between reading level and eccentricity was not significant $[F(3,36)=$ 1.72] when the analysis was confined to stimuli to the right of fixation; and the effect of reading level was not significant $[F(1,12)=1.3]$ when good and poor readers were compared at the most eccentric position in the right visual field (where performance was $47 \%$ and $61 \%$ correct for the good and poor readers, respectively). The results of these statistical tests provide no support for the view that within the right visual field we have replicated the Geiger and Lettvin (1987) and Perry et al. (1988, 1989) findings. Although acceptance of the null hypothesis is often a risky proposition, we believe that, in the present context, rejecting it on the basis of Geiger and Lettvin's (1987) and Perry et al.'s $(1988,1989)$ experiments is even riskier. In any event, even if the trend within the right visual field were reliable in our data, the absence of this trend in the other three directions (as well as in the overall results) demonstrates that the result reported by Geiger and Lettvin (1987) and Perry et al. (1988, 1989) is not due to different Aubert/Foerster functions in the good and poor readers.

Unlike Geiger and Lettvin (1987) and Perry et al. (1988, 1989), we did not find any difference in the acuity/ eccentricity function for good and poor readers. We believe that there is no difference, and that the pattern of results (dyslexics showing a broader Aubert-Foerster func-

Table 2

Percentage Correct for the Distance $\times$ Direction Interaction for Each Group in Both Experiments

\begin{tabular}{|c|c|c|c|c|c|}
\hline Eccentricity & Mean & Left & Right & $\mathrm{Up}$ & Down \\
\hline \multicolumn{6}{|c|}{ Good Readers-College } \\
\hline .7 & 96.6 & 97 & 98 & 95 & 96 \\
\hline 2.5 & 82.6 & 80 & 81 & 94 & 75 \\
\hline 5.0 & 65.9 & 78 & 72 & 51 & 62 \\
\hline 7.6 & 49.0 & 75 & 47 & 36 & 37 \\
\hline \multicolumn{6}{|c|}{ Poor Readers-College } \\
\hline .7 & 91.5 & 97 & 93 & 93 & 83 \\
\hline 2.5 & 75.0 & 71 & 73 & 83 & 72 \\
\hline 5.0 & 67.3 & 81 & 63 & 67 & 58 \\
\hline 7.6 & 52.7 & 83 & 61 & 29 & 37 \\
\hline \multicolumn{6}{|c|}{ Dyslexics-Adolescents } \\
\hline .7 & 91.0 & 91 & 92 & 94 & 85 \\
\hline 2.5 & 85.5 & 89 & 86 & 90 & 76 \\
\hline 5.0 & 61.9 & 66 & 67 & 58 & 47 \\
\hline 7.6 & 44.0 & 62 & 60 & 20 & 29 \\
\hline
\end{tabular}

tion) obtained by Geiger and Lettvin and by Perry et al. was artifactual, produced by a fixation (or attention) strategy difference between their good and poor readers. It might be argued, however, that our poor readers were not dyslexic or residually dyslexic, and that had we tested "true" dyslexic subjects our observations would have been the same as Geiger and Lettvin's and Perry et al.'s. Although both these studies used college students with reading difficulties as subjects (as we did), their criteria for classifying a student as dyslexic may have been more stringent than our criterion for classifying a student as a "poor" reader.

\section{EXPERIMENT 2}

We decided to administer the test described above to a group of teenagers who were residents at a school for children with severe reading problems. If we were correct, then these students should show the same pattern of results that we had already obtained for good and poor college readers. If, however, Geiger and Lettvin (1987) are correct, then with these "true" dyslexics we should find that the decline in accuracy with increasing eccentricity should be similar to the dyslexic function in Geiger and Lettvin (1987) and much flatter than that reported in Experiment 1.

\section{Method}

The apparatus used in Experiment 1 was transported to a residential school for reading disabled children, where a room was set aside for this research project. With the exception of subject selection and method of response, all procedures of this experiment were identical to those of Experiment 1. To avoid the frustration that our teenage subjects may have experienced trying to find the letters on the keyboard, we had them give their responses orally, with the experimenter entering the data via the keyboard.

Subjects. The 13 dyslexics ( 11 males and 2 females) who participated in the study were from a special school for the learning disabled. All were reading at least 2 years below grade level, and none had any record of hearing or visual loss or any other condition that could affect performance. All were of normal intelligence (as measured by the Wechsler Intelligence Scale for ChildrenRevised [WISC-R]), and in all but one case, the Performance Scale score on the WISC-R was higher than the Verbal Scale score. All information about these subjects was supplied by the school. Means, standard deviations, and ranges for age, IQ, and reading level (as measured by the Slosson Reading Test) and are given in Table 3 .

\section{Results and Discussion}

The results were subjected to a two-way ANOVA with direction and eccentricity as within-subject factors. The major finding was a main effect of eccentricity $[F(3,36)=$ $32.23, p<.001]$ (Figure 2). There was also a main effect of direction $[F(3,36)=9.05, p<.001]$, with more accurate performance in the left/right directions than in the up/down directions. This effect interacted with eccentricity; the decline in performance with increasing eccentricity was steeper in the up/down directions (see Table 2).

Visual comparison of the results of these dyslexic children with those of our good and poor college readers reveals that all three groups show a similar decline in identification accuracy as target eccentricity increases. To 
Table 3

Means, Standard Deviations, and Ranges for Age, Reading Level (Slosson), and IQ (WISC-R) of Dyslexic Subjects Used in Experiment 2

\begin{tabular}{lccc}
\hline & $M$ & $S D$ & Range \\
\hline Age & 15.8 & 1.67 & $13.10-18.10$ \\
Reading Level & 4.63 & 0.49 & $3.7-5.2$ \\
IQ (WISC-R) & & & \\
Full-Scale & 96.85 & 11.13 & $82-121$ \\
Performance & 103.84 & 15.04 & $78-130$ \\
Verbal & 91.00 & 9.57 & $77-109$ \\
\hline
\end{tabular}

confirm this, the data from the two experiments were subjected to a combined ANOVA with three groups (good college, poor college, dyslexic). This ANOVA revealed a significant effects of eccentricity $[F(3,72)=74.33, p<$ $.001]$ and direction $[F(3,72)=19.92, p<.001]$ and a significant interaction between these variables $[F(9,216)=$ $7.99, p<.001]$. Performance in the left and right directions is better than that in the up and down directions, primarily because the falloff with distance is much steeper for up and down. Neither the effect of group $[F(2,24)=$ .23 ] nor any interactions involving group [group $\times$ direction, $F(6,72)=.74$; group $\times$ eccentricity, $F(6,72)=1.4$; and group $\times$ direction $\times$ eccentricity, $F(18,218)=.83$ ] were significant.

\section{GENERAL DISCUSSION}

In the present study, we have demonstrated that there are no differences between good readers and poor readers or dyslexics in the pickup of peripheral information about letter identity. We would tentatively attribute the differences obtained by Geiger and Lettvin (1987) and Perry et al. $(1988,1989)$ to differences in attentional and/or fixation strategies. These proposed differences, of course, require explanation.

Our analysis (Footnote 1) shows that an optimal fixation strategy could easily have produced the dyslexic data pattern observed by Geiger and Lettvin (1987) and Perry et al. (1988, 1989, Experiment 2), and that suboptimal strategies could have produced the normal data pattern. Dyslexic individuals are acutely aware of their disability, and over the years have tried numerous strategies to overcome or conceal it. It seems likely that when confronted with the contingencies of the Geiger and Lettvin (1987) and Perry et al. $(1988,1989)$ task, the dyslexic readers were both better than the good readers at discerning the optimal strategy and perhaps less acquiescent about following the fixation instructions.

It might be argued that the Geiger and Lettvin (1987) pattern of results depends on the presence of the foveal letter, not because of differences in divided attention but because the foveal letter masks items in the periphery more (or at nearer distances) for normals than for dyslexics. Manipulation of the presence of the central letter is the obvious way to test this view of its role. Just such a manipulation was performed by Perry et al. (1988, 1989). They varied the peripheral target's eccentricity randomly (an improvement over Geiger and Lettvin's blocked procedure, even though they did not vary its direction) and between experiments compared the single (foveal or peripheral) and dual (foveal plus peripheral) target situations. Another positive aspect of their study was that all subjects in both conditions received the same exposure duration, a feature that permits comparisons across groups and experiments. Examination of their findings (Figures $1 B$ and $1 C$ ) reveals three points that are quite inconsistent with the notion that the Geiger and Lettvin pattern is due to differences between good and dyslexic readers in the degree of masking between foveal information and parafoveal information: (1) The dyslexics show flatter than normal functions in both the single and dual target conditions; (2) the dyslexic subjects suffer more than the normal readers at every eccentricity in the dual letter condition ${ }^{4}$; and (3) Perry et al.'s $(1988,1989)$ subjects' data are actually more similar to Geiger and Lettvin's (1987) in the single than in the dual target condition.

Geiger and Lettvin $(1987$, p. 1243) attribute their dyslexic performance pattern not merely to differences in peripheral vision, but to "an interaction between foveal and peripheral vision that degrades the ability to read in the foveal field." Thus, Geiger and Lettvin's proposal would appear to be that dyslexics have an improved pickup of information in peripheral vision, and that this has the effect of masking foveal information, thereby interfering with reading. Whether there are masking differences between good readers and dyslexics, as claimed by Geiger and Lettvin (1987), is not an issue that is directly addressed by the data presented here. However, by demonstrating that dyslexics and other poor readers do not have an improved pickup of information in peripheral vision, our study clearly undermines this proposal. We have recently obtained data suggesting that poor readers may have a broader "beam" of attention (D'Entremont \& Klein, 1989). When identifying a peripheral target in the presence of an irrelevant distractor at the same eccentricity as the target, poor readers are less affected by distractors near the attentional focus (i.e., close to the target) and more affected by distractors further from the attentional focus (i.e., distant from the target) than are good readers. Whether this finding is related to Geiger and Lettvin's proposal will be a topic for future research.

\section{REFERENCES}

D'Entremont, B., \& KLEIN, R. M. (1989). Selective attention and coding ability in good and poor readers. Unpublished manuscript.

Hughes, A. (1977). The topography of vision in mammals of contrasting life style: Comparative optics and retinal organization. In F. Crescitette (Ed.), Handbook of sensory physiology (The visual system in evolution. A. Vertebrates; Vol. VII/5, pp. 615-737). Berlin: SpringerVerlag.

Geiger, G., \& Lettuin, J. Y. (1987). Peripheral vision in persons with dyslexia. New England Journal of Medicine, 316, 1238-1243.

Perry, A. R., Dember, W. N., Warm, J. S., \& Sacks, J. G. (1988, November). Letter identification in normal and dyslexic readers: $A$ replication. Paper presented at the annual meeting of the Psychonomic Society, Chicago.

Perry, A. R., Dember, W. N., Warm, J. S., \& Sacks, J. G. (1989). Letter identification in normal and dyslexic readers: A verification. Bulletin of the Psychonomic Society, 27, 445-448. 
POSNER, M. I. (1980). Orienting of attention. Quarterly Journal of Experimental Psychology, 32, 3-25.

REED, A. V. (1979). Microcomputer display timing: Problems and solutions. Behavior Research Methods \& Instrumentation, 11, 572-576.

Shaywitz, B. A., \& Waxman, S. G. (1987). Dyslexia. New England Journal of Medicine, 316, 1260-1270.

WECHSLER, D. (1944). The measurement of adult intelligence (3rd ed.). Baltimore: Williams \& Wilkins.

WoODWORTH, R. S., \& SchlosBerg, H. (1954). Experimental psychology. New York: Rinehart \& Winston.

\section{NOTES}

1. The table below shows how correct letter identification would vary depending on location of fixation and location of letter. Line 1 (fixation at $0^{\circ}$ ) shows a hypothetical acuity/eccentricity function for Perry et al.'s $(1988,1989)$ experimental procedure. Given our assumption that the pickup of peripheral information is identical for normal and dyslexic readers, this function would be the same for both groups. Lines 2 and 3 show how performance would change for stimuli at the indicated locations if subjects fixated at $2.5^{\circ}$ or $3.75^{\circ}$ instead of at $0^{\circ}$.

Percent Letter Identification by Location of Fixation and Location of Letter

\begin{tabular}{lccccccc}
\hline \multirow{2}{*}{$\begin{array}{l}\text { Location of } \\
\text { fixation }\end{array}$} & $0^{\circ}$ & $1.25^{\circ}$ & $2.5^{\circ}$ & $3.75^{\circ}$ & $5.0^{\circ}$ & $6.25^{\circ}$ & $7.0^{\circ}$ \\
\hline$n^{\circ}$ & 95 & 93 & 90 & 80 & 60 & 30 \\
$2.5^{\circ}$ & 90 & & 95 & & 90 & 60 \\
$3.75^{\circ}$ & 80 & & 93 & & 93 & 80 \\
\hline
\end{tabular}

The Perry et al. pattern (Experiment 2; compare Figure 1C with Rows 2 and 3) can be quite closely simulated by supposing that their normal readers were fixating the $2.5^{\circ}$ position while their dyslexic readers were fixating the $3.75^{\circ}$ position. (Similar results can be obtained if subjects vary their gaze direction but adopt a mean position in line with the hypothetical locations given above.) The possibility that their readers might have been following such a strategy was discussed and rejected by Perry et al. (1989, p. 448). They correctly point out that this strategy predicts relatively uniform performance over a $5^{\circ}$ range for the dyslexic subjects (since that is what is shown in the normal data), but they err in implying that the data from their Experiment 2 are inconsistent with such a possibility.

2. Another technique for controlling fixation is to monitor eye position and reject trials on which a shift from fixation occurs. A combination of both procedures is ideal, but partly because we wanted to take this experiment on the road-where monitoring eye position would be unwieldy - we chose to eliminate the advantage subjects might derive from shifting gaze in the previous studies.

3. All the ANOVAs in this experiment were also conducted with gender as a between-subjects factor. There were no effects or interactions involving gender, so this variable was dropped in subsequent analyses.

4. Such a deficit could be due to (a) the mere presence of two items, even if only one was task relevant-that is, increased distractibility or "filtering cost" (see D'Entremont \& Klein, 1989); (b) the requirement to process information from two locations (i.e., divide or spread attention); (c) the requirement to perform two simultaneous identifications; and/or (d) hold information in memory while reporting the first.

(Manuscript received July 7, 1989;

revision accepted for publication December 8,1989 .) 